\section{EPILEPSY AND EEG}

\section{P.008}

Could Transient neurological Symptoms with subdural hematoma be explained by Cortical spreading depolarization Activity in Neurons? (CT-SCAN)

$M$ Levesque (Trois-Rivieres)* C Deacon (Sherbrooke) C Bocti (Sherbrooke) F Moreau (Sherbrooke) C Vézina (Sherbrooke) C IorioMorin (Sherbrooke)

doi: 10.1017/cjn.2018.110

Background: Transient neurological symptoms in patients with subdural hematoma (SDH) are often attributed to secondary epilepsy despite a negative workup. We believe a significant proportion of these patients could rather suffer from cortical spreading depolarization (CSD). Methods: We performed a retrospective case-control study of patients with transient neurological symptoms post-SDH evacuation between 1996 and 2017. The clinical features of patients with negative EEG were compared to those with positive EEG (ictal or interictal abnormalities) and a clinical scoring system was created. Results: 59 patients were included, 20 (34\%) with a positive EEG. Speech-related symptoms (OR 4.8, $\mathrm{p}=0.018$ ) and prolonged episodes (OR 23.1, $\mathrm{p}=0.001$ ) were associated with a negative EEG. Clonic movements (OR 0.014, $\mathrm{p}<0.0005$ ), impaired awareness (OR $0.013, \mathrm{p}<0.0005$ ), positive symptoms (OR $0.05, \mathrm{p}<0.0001$ ), complete response to standard antiepileptic drugs (OR 0.06, $\mathrm{p}=0.007$ ) and mortality (OR 0.021, p=0.003) were associated with a positive EEG. We built a clinical score based on these features, which showed a $90 \%$ sensitivity and $100 \%$ specificity. Conclusions: We believe that the differences observed between both groups were driven by the presence of CSD rather than seizure in the case group. Our proposed scoring system can help predict EEG results and may be useful to identify CSD in future trials.

\section{P.009}

\section{Improving triaging of Infantile Spasm EEG referrals in a large Canadian institution}

D Djordjevic (Toronto)* C Go (Toronto)

doi: 10.1017/cjn.2018.111

Background: Infantile spasms (IS) is a seizure disorder in young children which can be challenging to recognize. Early diagnosis and treatment of IS is critical for prevention of developmental delay. At the Hospital for Sick Children, rule-out IS accounts a large percentage of outpatient EEG referrals, while few result in a diagnosis. The goal of this study was to characterize the seizure semiology and source of referral, in order to explore ways in which triaging of these referrals may be improved. Methods: A retrospective review was done on all 84 rule-out IS EEG referrals within the last year at the Hospital for Sick Children. Source of referral, description of episodes, and result of EEG was extracted and data qualitatively analyzed. Results: Neurologists at Hospital for Sick Children accounted for the least number of referrals however contributed the greatest percentage of IS diagnoses. Non-neurologists contributed the most referrals, however $75 \%$ of these EEGs were normal. Common semiologies as described by referring physicians are discussed. Conclusions: Improved triaging of EEG referrals may be achieved through screening of referrals by paediatric neurologists, clarification of event semiology, and better IS education. This represents a critical opportunity for improvement of resource allocation as well as patient care and outcomes within all major referral centres.

\section{P.011}

Not just for babies: positive rolandic sharp waves in adult post-hypoxic myoclonus

GA McLeod (Winnipeg)* MC Ng (Winnipeg)

doi: 10.1017/cjn.2018.113

Background: Post-hypoxic myoclonus is broadly divided into myoclonic status epilepticus (MSE) and Lance-Adams syndrome (LAS), where diagnosis depends on clinical and electroencephalographic (EEG) findings. Positive rolandic sharp waves (PRS) are a classic EEG finding in pre-term infants with white matter necrosis, but they are not known to be epileptogenic and have never been described in adults. Methods: We report a unique case of PRS correlated with myoclonic seizures in a post-hypoxic adult patient. Results: Shortly after cardiac arrest, a 21-year-old woman developed multifocal post-hypoxic myoclonus. Early development of myoclonus suggested MSE, but her EEG findings were atypical for MSE; initially, the only notable feature on EEG were subtle PRS. LAS did not fit the clinical picture or EEG findings. As myoclonus persisted over the following weeks, PRS evolved on EEG into positive rolandic predominant generalized polyspike-wave complexes that became definitively time-locked to each myoclonic jerk. PRS were diagnosed as epileptogenic and frequent myoclonic jerks were diagnosed as continuous myoclonic seizure. Myoclonus resolved to medication and mental status returned to baseline. Conclusions: We report for the first time that PRS can appear in adult patients and be epileptogenic, and produce a non-classical variant of post-hypoxic myoclonus that carries good prognosis.

\section{P.012}

Post-operative hippocampal volume changes on magnetic resonance volumetry in patients with mesial temporal lobe epilepsy

K Iida (Hiroshima)* J Katayama (Hiroshima) K Kagawa (Hiroshima) M Katagiri (Cleveland) G Seyama (Hiroshima) A Hashizume (Hiroshima) K Kurisu (Hiroshima) H Otsubo (Toronto)

\section{doi: 10.1017/cjn.2018.114}

Background: We evaluate long-term post-operative hippocampal volume (HV) on non-epileptic hippocampus using MR volumetry as well as the neuropsychological outcome in patients with surgery for unilateral mesial temporal lobe epilepsy (MTLE) and achieved seizure-freedom. Methods: We studied 1.5-Tesla MRI before and after epilepsy surgery in 24 patients with MTLE. Serial MRI studies were scheduled at 4 post-operative consecutive periods; $6 \mathrm{~m}-1 \mathrm{y}$; 1-2y; 2-3y; 3-5y. We compared neuropsychological outcomes for memory and estimated IQ at the same periods with serial MRI up to 3 years. Results: The pre-operative non-epileptic HV was significantly smaller than HV in age-matched controls $(n=14)(p<0.05)$. The HV became progressively atrophic after the surgery $(\mathrm{p}<0.05)$, correlating with the age at surgery $(\mathrm{p}<0.05)$ and pre-operative larger non-epileptic HV $(\mathrm{p}<0.05)$, but not with seizure duration. In 14 patients with 
non-dominant MTLE, the smaller dominant HV at 2-3y period correlated with decline of verbal memory $(\mathrm{p}<0.05)$. Conclusions: Postoperative progression of non-epileptic hippocampal atrophy was found with significantly more pronounce in patients with older age at surgery and larger pre-operative non-epileptic hippocampus. After the epileptogenic hippocampus is resected, the remaining hippocampus alone might exhaust to maintain the memory, especially in elders.

\section{P.013}

Convulsive status epilepticus due to intracranial hypotension GS Gilmour (Calgary) J Scott (Calgary) P Couillard (Calgary)*

doi: 10.1017/cjn.2018.115

Background: Intracranial hypotension (IH) is typically characterized by an orthostatic headache. There have been limited case reports describing iatrogenic IH presenting with seizures. Methods: Case report. Results: A 71-year-old woman with chronic back pain developed convulsive status epilepticus (SE), characterized by generalized clonic seizures, immediately following scoliosis surgery. She had no history of seizures or seizure risk factors. Despite treatment with Midazolam, Phenytoin and Lacosamide, seizures recurred five times over three hours. Thus, Propofol and Midazolam infusions were initiated. An electroencephalogram revealed burst suppression and bilateral hemispheric epileptiform discharges. MRI brain was consistent with $\mathrm{IH}$ without cortical vein thrombosis. Fluid from the surgical drains was positive for Beta-2 transferrin, indicating cerebral spinal fluid. Her intracranial hypotension was likely due to an intraoperative dural tear causing SE. Over two weeks, she remained on bedrest, sedation was weaned, and Phenytoin and Lacosamide were tapered and discontinued. She had no further seizures. Conclusions: IH is an under recognized cause of seizure following spinal or cranial surgery, lumbar puncture, or spinal anaesthesia. Proposed mechanisms include traction on cortical structures, increased cerebral blood flow and cortical irritation secondary to subdural hygromas.

\section{P.014}

Survey of epilepsy and seizure awareness in Manitoba: an evaluation (SESAME)

\section{EC Avery (Winnipeg) C MacDonald (Winnipeg) M Ng (Winnipeg)* $D$ Serletis (Winnipeg)}

doi: 10.1017/cjn.2018.116

Background: Epilepsy/seizure awareness is improving across Canada. With the formation of a Comprehensive Epilepsy Program in Manitoba (including a new Pediatric Epilepsy Monitoring Unit), a provincial strategy has been proposed outlining a path towards improved access to epilepsy care. We now sought to qualify the current state of clinician knowledge and comfort towards diagnosis and management of this condition. Methods: A qualitative online survey, comprised of 36 short-answer questions, was delivered to primary care and specialist physicians in Manitoba. Results:108 subjects responded, across varying medical disciplines. 101 (93.5\%) have previously managed epilepsy patients, and 87 (80.6\%) have previously ordered an EEG. A total of 63 (59.4\%) have referred to a neurologist, with a lower proportion (30, 28.3\%) referring specifically to an epileptologist. 36 respondents (33.3\%) have heard of the ILAE guidelines, with 43 (63.2\%) reporting refractory epilepsy to be defined by the failure of 3 (or more) medications. 61 (56.5\%) were unaware of invasive EEG techniques. Most $(85,78.7 \%)$ understood a role for surgery in treating epilepsy, with 12 (11.1\%) unaware of surgical therapies beyond VNS. Conclusions: SESAME successfully identified strong awareness towards epilepsy, with small lapses in knowledge that will benefit from a formal provincial-wide educational curriculum.

\section{P.015}

Mesial Temporal Sclerosis is a rare occurrence in Intractable Pediatric Temporal Lobe Epilepsies

J Kassiri (Edmonton)* T Rajapakse (Edmonton) L Schmitt (Edmonton) $M$ Wheatley (Edmonton) B Sinclair (Edmonton)

doi: 10.1017/cjn.2018.117

Background: Temporal lobe epilepsy (TLE) accounts for approximately $20 \%$ of pediatric epilepsy cases. Of those, many are considered medically intractable and require surgical interventions. In this study, we hypothesized that mesial temporal sclerosis (MTS) was less common in patients who had undergone surgery for intractable pediatric TLE than in adult series. We further hypothesized that there was a radiological and pathological discordance in identifying the cause of pediatric TLE. Methods: We retrospectively reviewed the charts of pediatric patients with TLE who had undergone surgical treatments as part of the University of Alberta's Comprehensive Epilepsy Program between 1988 and 2018. Along with preoperative magnetic resonance imaging (MRI) reports, post-surgical pathology results and seizure outcomes were studied Results: Of the 83 pediatric patients who had undergone temporal lobe epilepsy surgery, $28 \%$ had tumors, $22 \%$ had dual pathologies, $18 \%$ had MTS, $11 \%$ had focal cortical dysplasia, and $22 \%$ had other pathologies. In addition, for $36 \%$ of these patients, discordance between their pre-surgical MRI reports and post-surgical pathology reports were found. Conclusions: This was one of the largest retrospective cohort studies of pediatric patients who had undergone surgery for intractable TLE. This study showed that tumors, and not MTS, were the most common pathology in surgical pediatric TLE .

\section{P.016}

A novel de novo GABRA1 mutation linked to epileptic encephalopathy: pathophysiology and potential therapeutic options

$M$ Chiu (Vancouver)* Y Bai (Vancouver) EH Chan (Vancouver) $L$ Huh (Vancouver) I Guella (Vancouver) MJ Farrer (Vancouver) $M$ Connolly (Vancouver) L Liu (Vancouver) M Demos (Vancouver) $Y$ Wang (Vancouver)

doi: 10.1017/cjn.2018.118

Background: Epileptic encephalopathy (EE) is a severe neurological disorder characterized by treatment-resistant seizures and poor neurodevelopmental outcomes. EE is associated with mutant genes, including those that encode for $\gamma$-aminobutyric acid type A (GABA) receptor subunits. We identified a novel de novo GABRA1 mutation in a patient with EE, characterized its impact on GABA receptor function, and sought potential therapeutic options. Methods: We described the clinical and electrophysiological features of a patient with a novel de novo GABRA1 (R214C) mutation; performed 